\title{
LETTERS
}

\section{Tools to improve reporting of patient-oriented research}

Our collaborative research team commends CMAJ for launching the patientoriented research collection in CMAJ Open. ${ }^{1}$ Particularly, the specification for authors to report "how patients were engaged as part of the research team and how their contributions developed the work" is an opportunity to gather rich information about the role and function of patient partners. ${ }^{1}$

At the recent Knowledge Translation (KT) Canada Scientific Meeting 2018 (https:// ktcanada.org/event/kt-canada-scientific -meeting-2018/), there was ample discussion about the lack of guidelines to report patient engagement in research. We believe research articles should consistently provide detailed yet concise information about the engagement of patients in research projects. Indeed such information, when gathered by systematic reviews, could inform methodology for best practices of patient-oriented research.

An international effort has created the second version of the Guidance for Reporting Involvement of Patients and the Public (GRIPP2) checklist to facilitate standardization for studies to report patient and public engagement in research in publications. ${ }^{2}$ Having recognized the low-quality reporting of patient engagement in research, our research team has also published a framework outlining the core components for describing the processes of patient engagement. ${ }^{3}$ Our framework complements the GRIPP2 and provides additional guidance for authors to consider while reporting the details of patient engagement in research studies. For example, for the methods section, the GRIPP2 recommends clear description of the engagement methods, whereas our framework provides possible scope and descriptors. Contributors to CMAJ Open could use these valuable tools when preparing their manuscript for the patient-oriented research collection.

\section{Clayon B. Hamilton PhD}

Postdoctoral research fellow, Department of Physical Therapy, University of British Columbia, Vancouver, BC; postdoctoral trainee, Arthritis Research Canada, Richmond, BC

\section{Alison M. Hoens MSc BScPT}

Member of Arthritis Patient Advisory Board, Arthritis Research Canada, Richmond, BC; clinical professor, Department of Physical Therapy, University of British Columbia, Vancouver, BC

\section{Linda C. Li PhD PT}

Professor, Department of Physical

Therapy, University of British Columbia, Vancouver, BC; senior scientist, Arthritis Research Canada, Richmond, BC

Cite as: CMAJ 2018 October 15;190:

E1234. doi: 10.1503/cmaj.70187

\section{References}

1. Patrick K, Kebbe M, Aubin D. A home for patientoriented research. CMAJ 2018;190:E607.

2. Staniszewska S, Brett J, Simera I, et al. GRIPP2 reporting checklists: tools to improve reporting of patient and public involvement in research. BMJ 2017;358:j3453.

3. Hamilton CB, Leese JC, Hoens AM, et al. Framework for advancing the reporting of patient engagement in rheumatology research projects. Curr Rheumatol Rep 2017;19:38.

Competing interests: None declared. 